\title{
Impact of granular drops
}

\author{
J. O. Marston, M. M. Mansoor, and S. T. Thoroddsen \\ Division of Physical Sciences and Engineering, King Abdullah University of Science and Technology, Thuwal 23955-6900, Saudi Arabia
}

(Received 20 April 2013; published 15 July 2013)

\begin{abstract}
We investigate the spreading and splashing of granular drops during impact with a solid target. The granular drops are formed from roughly spherical balls of sand mixed with water, which is used as a binder to hold the ball together during free-fall. We measure the instantaneous spread diameter for different impact speeds and find that the normalized spread diameter $d / D$ grows as $(t V / D)^{1 / 2}$. The speeds of the grains ejected during the "splash" are measured and they rarely exceed twice that of the impact speed.
\end{abstract}

DOI: 10.1103/PhysRevE.88.010201

PACS number(s): $83.80 . \mathrm{Fg}$

The flow and qualitative behavior of granular material in various situations can be truly fluidlike [1]. This has become evident through experimental studies with dry, loose granular materials, where interparticle collisions and friction tend to govern the observed dynamics. For solid sphere impact onto a static bed of fine powders, one can observe ejecta sheets at short time scales [2] and granular jets following the collapse of the cavity behind the sphere [3-7]. For a sphere falling through ultra-low-density grains, a terminal velocity has also been observed [8], while other flow configurations present a direct analogy to experiments with liquids such as the rupture of a granular jet [9], the granular jump [10,11], fingering patterns $[12,13]$, and the granular equivalent of a water bell [14].

In this Rapid Communication, we build on this theme of "granular equivalent" experiments by examining the impact of granular drops, which are roughly spherical balls of granular material with a small water content. In particular, we seek to determine if the temporal evolution of the spreading can be related to that observed during the impact of pure liquid drops. We also compare the velocities of grains which are ejected in the "splash" to those ejected from dry granular beds during solid-body impact. While one would intuitively expect some differences between the impact of liquid drops and granular drops due to dissipation mechanisms (e.g., viscous and surface tension forces vs interparticle collisions) and for dry vs wet granular media due to added dissipation through liquid bridges, we find some subtle similarities in both regards.

Figure 1 shows select frames from high-speed video sequences for granular and water drops, both impacting the target substrate with velocity $V \approx 2.6 \mathrm{~m} / \mathrm{s}$. The corresponding times from first contact are shown in each frame. We note the rapidity of the dynamics in the (incompressible) water drop, whereby the ejecta sheet is seen $100 \mu$ s after contact and the first detectable satellite drops emerge as early as 600 $\mu \mathrm{s}$, at which point the drop has spread approximately two initial drop diameters $(d / D \approx 2)$. In contrast, for the granular drop, no individual grains are ejected until approximately $3 \mathrm{~ms}$ from first contact, whereupon the drop has already spread one diameter $(d \approx D)$. Note, however, that the scaled times for the emergence of the first drops in the liquid case, $t V / D=0.43$, and the first grains in the granular case, $t V / D=0.3$, are very similar.

Methods. To make granular drops, we use a commercially available granular material (Ottowa sand, Fisher Scientific) with mean grain size $d_{g}=350 \mu \mathrm{m}$, grain density $\rho_{g}=$ $2.65 \mathrm{~g} / \mathrm{cm}^{-3}$, and mean circularity $C=4 \pi A / P^{2}=0.81$, where $A$ and $P$ represent the area and perimeter of the grains determined from two-dimensional microscope images. The dry material is then mixed with water $(10 \%$ water content by mass) to form the wet granular material, which can be molded into approximately spherical balls by scooping an overrequired amount of material with one hemispherical spoon and then compressing the wet material between two identical hemispherical spoons $26 \mathrm{~mm}$ in diameter. This particular water content was chosen crudely as the minimum required to achieve cohesion. The balls have a mass of approximately $15 \mathrm{~g}$ and volume of $9.2 \mathrm{~cm}^{3}$, yielding solids volume fractions, $\phi \approx 0.55$, equivalent to a saturation of $30 \%$, thus implying the liquid is in the form of liquid bridges throughout. One spoon is then removed and the other is used to hold the wet granular ball before lightly tapping to promote the ball's release from a known height $h$ above the target surface. The granular ball then falls freely under gravity to impact a $2 \mathrm{~cm}$ thick target glass plate, measuring $40 \times 40 \mathrm{~cm}$, with impact speed $V \approx \sqrt{2 g h}$. The target plate is cleaned between each trial.

We capture the impact event using a high-speed video camera (Photron Fastcam SA-5) operating at $10000 \mathrm{fps}$ with both back and front illumination. For both granular and water drops, the impact speed $V$ is measured directly from the acquired video sequence along with the instantaneous spread diameter $d$. For granular drops, we also perform particle tracking in order to obtain velocities of grains that are ejected during impact, while for liquid drops, we manually track satellite drops to obtain their velocities. The selection of a Nikon Micro-Nikkor $105 \mathrm{~mm}$ lens and wide aperture renders a narrow depth of field so that the measurement plane is established optically (through the center of the drop) and particles with out-of-plane motion are easily discounted.

Spreading. Figures 2(a) and 2(b) show the spread diameter $d$ measured along the surface of the target plate, and height $h$ of the top of the granular drop measured vertically from the plate surface, respectively, against time from impact. In Fig. 2(a), we find true maximum spread diameters only for the lowest impact speed since all other impacts result in granular matter being ejected out of the field of view, with discrete clumps forming. Nonetheless, we can clearly see that the spread rate increases monotonically with impact speed, as one would expect. The same trend is observed for the decrease in height $h$, shown in Fig. 2(b); here the drop height decreases from $h \approx 26 \mathrm{~mm}$ 

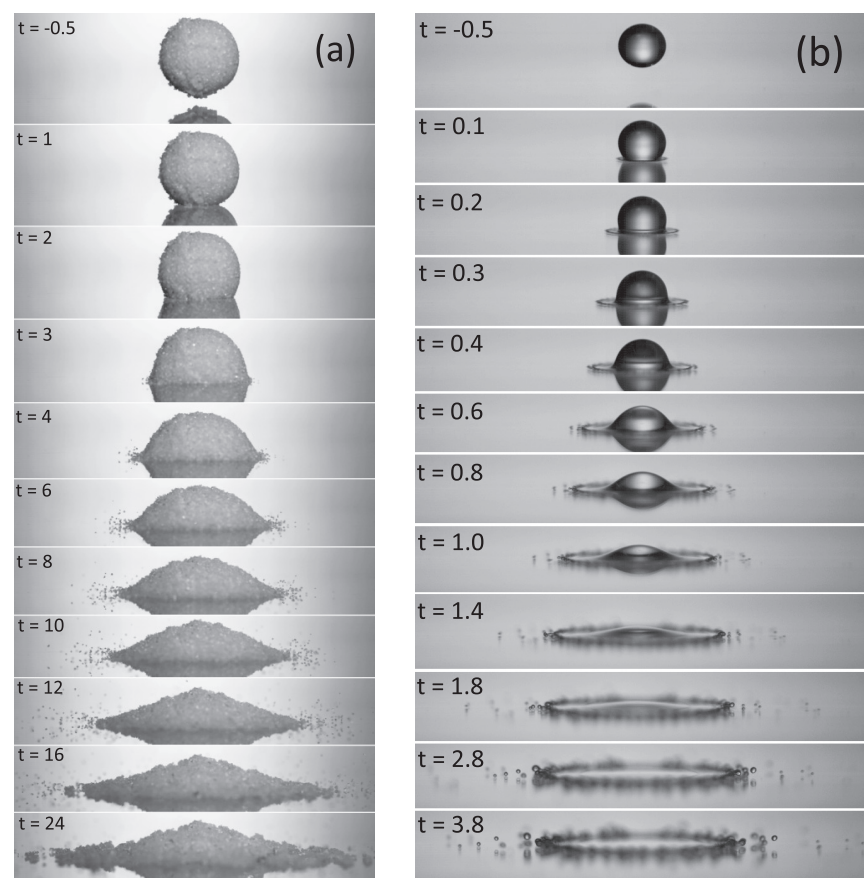

FIG. 1. Impact of (a) a $26 \mathrm{~mm}$ granular drop onto a thick glass plate and (b) a $3.6 \mathrm{~mm}$ water droplet onto a hydrophobic glass plate. The times shown are in milliseconds. The impact speed in both cases is $V \approx 2.6 \mathrm{~m} / \mathrm{s}$.

(i.e., one drop diameter) to some minimum level $h_{\min }=10-16$ $\mathrm{mm}$ reached approximately $15 \mathrm{~ms}$ after impact, after which no further changed is observed.

Using the natural length scale $D$ and time scale $D / V$ for this phenomenon, we can normalize the data in Figs. 2(a) and 2(b) to render the scaled plots shown in Figs. 2(c) and $2(\mathrm{~d})$ for $\beta=d / D$ versus $T=t V / D$, and $H=h / D$
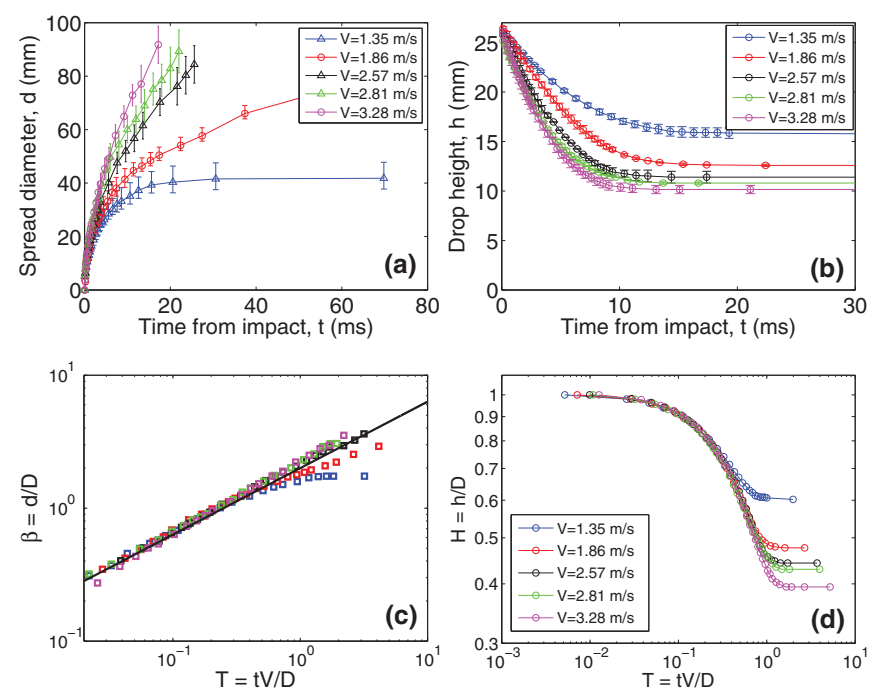

FIG. 2. (Color online) Maximum spread (a) and drop height (b) versus time. Scaled data are shown in (c) and (d), respectively, where the spread diameters and heights are normalized by the initial drop diameter $D$, and time is normalized by the timescale $D / V$. The solid black line in (c) indicates a slope of $1 / 2$. Error bars encompass multiple trials [not shown in (c) and (d)]. versus $T$, respectively. In Fig. 2(c), we find a remarkably similar scaling to the spreading of pure water drops, whereby $\beta \sim T^{1 / 2}$, which was previously observed in experiments [15] and theoretical studies [16]. This scaling, which collapses our data, corresponds to self-similar expansion and appears to hold for early times with $T<1$, after which grains are ejected where the data sets shown in Fig. 2(c) begin to diverge.

In contrast, however, the scaled height $H$ exhibits no such power-law dependence on $T$. This is evident from the log-log plot shown in Fig. 2(d). Rather, we observe a consistent linear decay in $H$ for $T \leqslant 0.3$, whereby $H \approx 1-0.9 T$ for all impact speeds shown. From simple geometrical arguments for $T<1$, considering a sphere moving at constant speed $V$ intersecting a plane (neglecting the ejected material or internal compression), one would expect the spread diameter to follow a $T^{1 / 2}$ scaling and the height to follow a $\Delta H=-T$ decay, which is not a bad approximation for the early evolution here.

Unlike pure liquid drops where the apex height continues to diminish down to $h \approx 0.01 D$ and can even de-wet the surface [17], our granular drops reach a minimum height, manifested as a clear peak [see final images in Fig. 1(a)], whereas liquid drops continue to deform (down to $h / D<0.1$ ) as the drop spreads radially outward. Note also that the granular drops used herein are compressible, so that volume is not conserved during the initial spreading phase (between initial contact and grain ejection). To highlight this important point, we present images in Fig. 3 of the drop just prior to impact and just before grains are ejected, where the boundaries of the shapes are highlighted in red (or dark gray). Using simple image analysis, we then estimate the respective volumes of the granular drops at each stage for the whole range of impact speeds. We find, as noted above, that the mean solids packing in the drops is $\phi \approx 0.55$, based on the volume and water content. From the shape analysis such as that in Fig. 3, we then find that the mean change in packing fraction is $\Delta \phi \approx 0.08$, meaning that the solids packing just prior to impact is approximately $\phi=0.63$, close to the random close packing limit. It is when this limit is approached, which coincides with a spread diameter $d \approx D$, that grain ejection occurs.

It is also interesting to note here that in experiments involving the impact of a solid sphere onto granular beds, it was found that grains were ejected only after the sphere had penetrated a small distance into the bed, while the early temporal evolution of the ejecta neck also followed an approximate square-root dependence on time [2].

Grain ejection velocities. An example of the particle tracking analysis is shown in Fig. 4(a) for times $t=-0.4$,
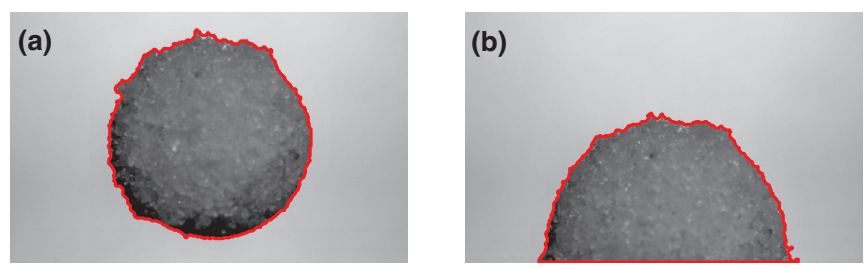

FIG. 3. (Color online) Images with highlighted boundaries (a) just before impact and (b) just before grain ejection. The change in area inside the boundaries determines the change in solids packing during impact. 
(a)

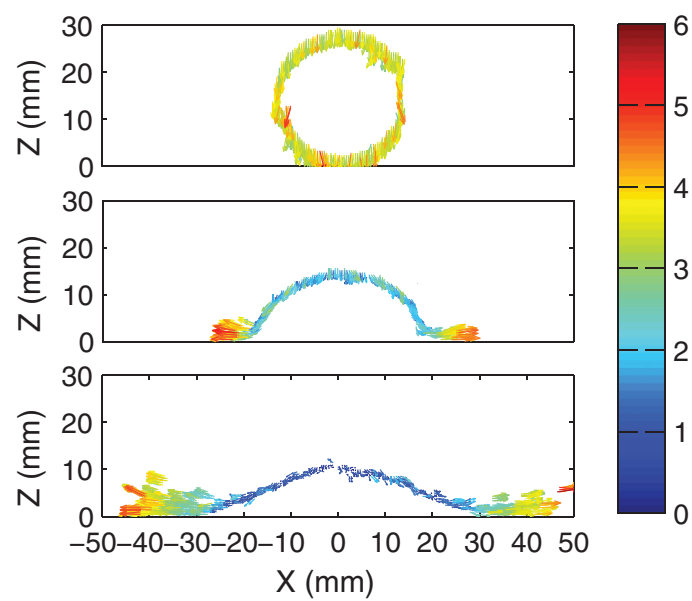

(b)

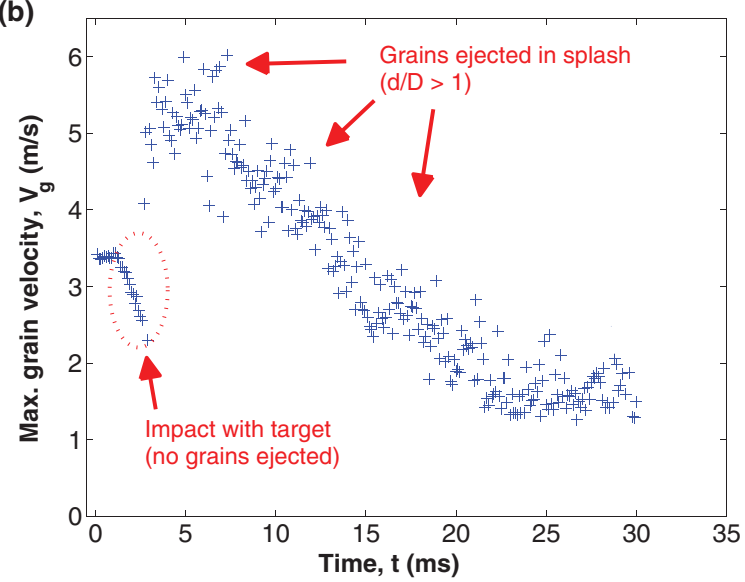

FIG. 4. (Color online) (a) Snapshots of the velocity field rendered after particle tracking, taken at times $t=-0.4,4.5$, and $9.5 \mathrm{~ms}$ from impact. $D=26 \mathrm{~mm}, V=3.3 \mathrm{~m} / \mathrm{s}$. (b) Maximum instantaneous particle velocity versus time from impact.

4.5, and $9.5 \mathrm{~ms}$ from first contact, whereby only particles on the edge of the drop are detected during the initial collision with the target, but individual grains are found during the splash. Since we are interested in determining the speed of the fastest grains ejected in this process, we plot the corresponding maximum particle velocity found anywhere in the domain at each time step in Fig. 4(b) where, for $t<3 \mathrm{~ms}$, the data points clearly correspond to particles residing at the top of the drop undergoing deceleration due to the collision. As grains are ejected in the splash for $d / D>1$ [e.g., second image in Fig. 4(a)], the peak velocities are attained, as shown by the sharp jump in $V_{g}$ in Fig. 4(b). The grain velocities then appear to decay linearly with time. Although we can only track grains residing at the edge of the drop or individual grains ejected in the splash, the maximum velocities found are expected to represent the true maximum since grains in the interior are constrained both by other grains in contact and by liquid bridges, which also act as a form of dissipation during impact. We do not expect air drag to play a significant role since the effective distance over which air drag becomes important $L_{\text {drag }}=4 d_{b} \rho_{g} / 6 \rho_{\text {air }} \approx 50 \mathrm{~cm}$ for these grains, thus the decay in ejection velocity with time is inherently determined by the dynamics of the impact itself, in particular the deceleration of the central part of the drop and the dissipation of kinetic energy via compression and liquid bridges. We also observe fracturing during the latter stages of impact and the formation of clumps, restricting the frequency of individual grain ejection.

For impact speeds $V \geqslant 1.84 \mathrm{~m} / \mathrm{s}$, we find that the fastest grains emerge with velocities approximately twice that of the impact speed, where the ratio $V_{g} / V=2.1-2.3$ for all impact speeds, followed by a decay of ejection velocity in time, as shown by Fig. 5(a), where we plot velocity-time data averaged over repeat trials for fixed impact speeds (shown in the legends), and Fig. 5(b), where we plot the maximum grain velocity versus drop impact speed. For the two highest impact speeds, we observe sharper decay of velocity with time compared to the lower two impact speeds, due to the higher initial velocities in the splash. In all cases, however, the initial impact is well characterized by the deceleration phase, followed by a marked jump to the maximum velocity as the first grains are ejected.

During solid sphere impact onto dry granular beds, the fastest grains were found to emerge with speeds up to 5 times that of the impactor, i.e., $V_{g} / V=5$, while the decay of grain
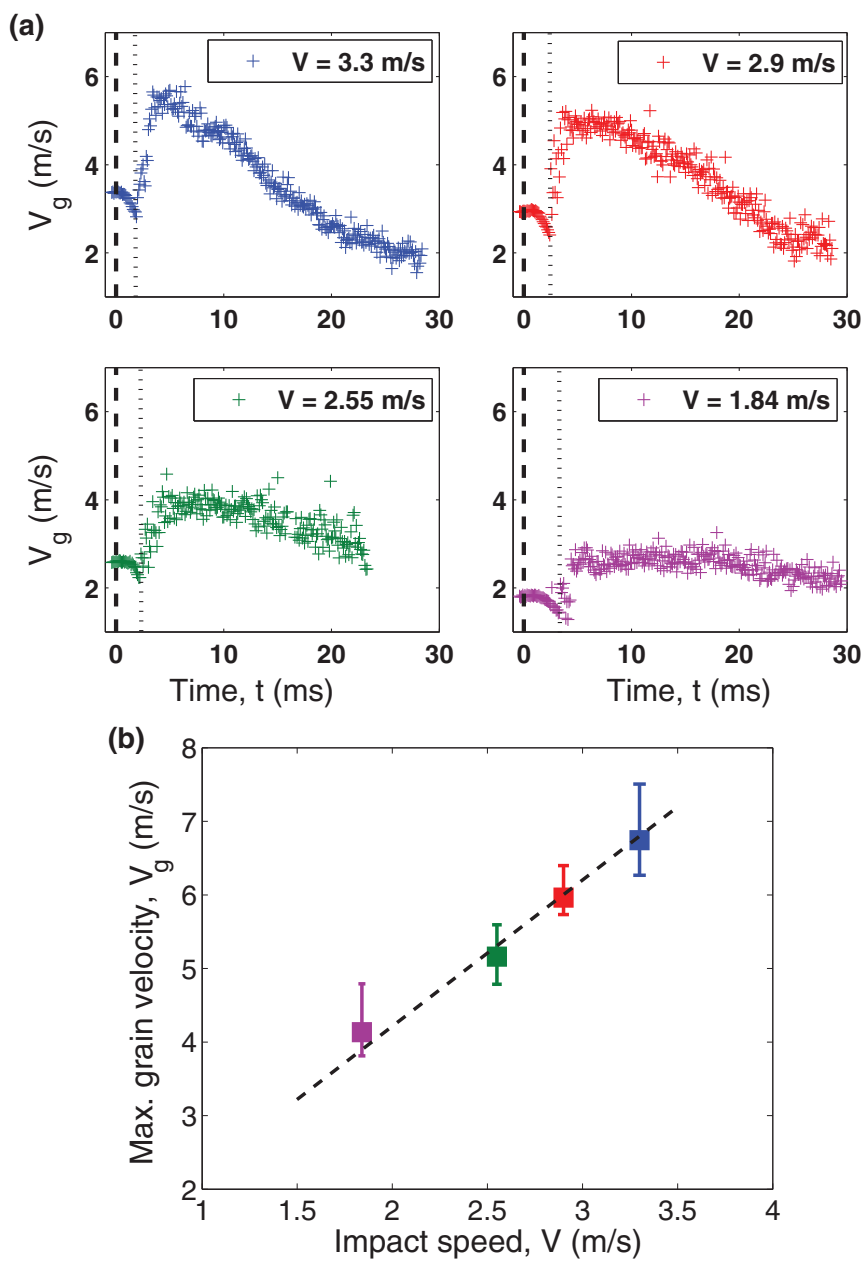

FIG. 5. (Color online) (a) Peak grain velocities versus time from impact. Data shown in each plot is averaged over five different realizations. The vertical lines indicate the moment of first contact (dashed) and first ejection of grains (dotted). (b) Maximum grain velocity versus impact speed. The dashed line indicates a slope of 2 . 
ejection speeds with time followed a simple power law scaling with $V_{g} / V \sim T^{-1}$ [2]. In contrast, for satellite drops ejected during the splash of liquid drops onto solid substrates, the ejection speeds were found to fit $V_{\text {sat }} \sim t^{-1 / 2}$ [18]. Discrete grains from the wet granular balls must first overcome liquid bridges which initially hold the particles together, this would clearly then limit the ejection velocity in comparison to dry material [as is the case for impacts onto loose (dry) granular beds], which thus implies that the liquid binder properties, notably the viscosity, must influence the grain ejection speeds. Of particular interest is the study in [19], which deduced that viscous dissipation in liquid bridges can account for up to $97 \%$ of the energy loss during low-speed $(V<6 \mathrm{~m} / \mathrm{s})$ impact of small wet granules.

Concluding remarks. In this Rapid Communication, we have studied the impact of granular drops onto solid target surfaces. By measuring the spread diameter versus time and scaling with the natural length and time scales, we find a remarkable similarity between granular drops and pure water droplets whereby both exhibit initial growth as $T^{1 / 2}$. This finding would appear to further strengthen the analogy between pure fluid and granular systems. In contrast, however, the height of the apex of the drop decays almost linearly with time for $T<0.3$, then gradually transitions to a minimum height, manifested by a distinct peak. We have also measured the velocities of individual grains ejected during the splash and found that the maximum velocities rarely exceed twice that of the impact velocity, $V_{g} / V \leqslant 2$, whereas in impacts onto dry granular beds, $V_{g} / V \leqslant 5$, and for pure water drops the satellite droplets can attain high speed with $V_{\text {sat }} / V \approx 20$. Clearly, there are many possible iterations of the experiment reported here, including varying the liquid binder viscosity and content. In addition, another logical step would be to assess granular ball impact using dried balls as used in [20], rather than those formed with liquid binder, to compare the ejection speeds between wet and dry balls. These parameters are the subject of ongoing investigation and will be addressed in future publications. Experiments such as those described herein and in [21] also serve as a first step to elucidate the early-time dynamical response of wet granular materials to impact phenomena, which is of general interest due to the ubiquity of wet granular materials in nature.
[1] J. R. de Bruyn, J. Fluid Mech. 704, 1 (2012).

[2] J. O. Marston, E. Q. Li, and S. T. Thoroddsen, J. Fluid Mech. 704, 5 (2012).

[3] S. T. Thoroddsen and A. Q. Shen, Phys. Fluids 13, 4 (2001).

[4] D. Lohse, R. Bergmann, R. Mikkelsen, C. Zeilstra et al., Phys. Rev. Lett. 93, 198003 (2004).

[5] D. Lohse, R. Rauhe, R. Bergmann, and D. van der Meer, Nature 432, 689 (2004).

[6] J. R. Royer, E. I. Corwin, A. Flior, M.-L. Cordero, M. L. Rivers, P. J. Eng, and H. M. Jaeger, Nat. Phys. 1, 164 (2005).

[7] J. O. Marston, J. P. K. Seville, Y-V. Cheun, A. Ingram, S. P. Decent, and M. J. H. Simmons, Phys. Fluids 20, 023301 (2008).

[8] F. Pacheco-Vázquez, G. A. Caballero-Robledo, J. M. SolanoAltamirano, E. Altshuler, A. J. Batista-Leyva, and J. C. RuizSuárez, Phys. Rev. Lett. 106, 218001 (2011).

[9] J. R. Royer, D. J. Evans, L. Oyarte, Q. Guo et al., Nature 459, 1110 (2009).

[10] J. F. Boudet, Y. Amarouchene, and H. Kellay, J. Fluid Mech. 572, 413 (2007).
[11] C. G. Johnson and J. M. N. T. Gray, J. Fluid Mech. 675, 87 (2011).

[12] O. Pouliquen, J. Delour, and S. B. Savage, Nature 386, 816 (1997).

[13] X. Cheng, L. Xu, A. Patterson, H. M. Jaeger, and S. R. Nagel, Nat. Phys. 4, 234 (2008).

[14] X. Cheng, G. Varas, D. Citron, H. M. Jaeger, and S. R. Nagel, Phys. Rev. Lett. 99, 188001 (2007).

[15] R. Rioboo, M. Marengo, and C. Tropea, Exp. Fluids 33, 112 (2002).

[16] J. Eggers, M. A. Fontelos, C. Josserand, and S. Zaleski, Phys. Fluids 22, 062101 (2010).

[17] J. E. Sprittles and Y. D. Shikhmurzaev, Phys. Fluids 24, 082001 (2012).

[18] S. T. Thoroddsen, K. Takehara, and T. G. Etoh, J. Fluid Mech. 706, 560 (2012).

[19] J. Fu, M. J. Adams, G. K. Reynolds, A. D. Salman, and M. J. Hounslow, Powder Tech. 140, 248 (2004).

[20] F. Pacheco-Vazquez and J. C. Ruiz-Suarez, Phys. Rev. Lett. 107, 218001 (2011).

[21] J. O. Marston, I. U. Vakarelski, and S. T. Thoroddsen, Phys. Rev. E 86, 020301 (2012). 